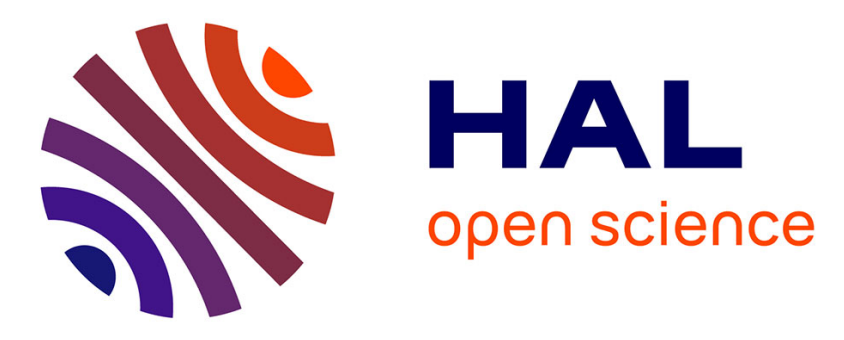

\title{
First Step in Cross-Layers Measurement in Wireless Networks How to Adapt to Resource Constraints for Optimizing End-to-End Services?
}

Philippe Owezarski, Rasha Ghassan Hasan, Guillaume Kremer, Pascal Berthou

\section{To cite this version:}

Philippe Owezarski, Rasha Ghassan Hasan, Guillaume Kremer, Pascal Berthou. First Step in CrossLayers Measurement in Wireless Networks How to Adapt to Resource Constraints for Optimizing End-to-End Services?. 9th Wired/Wireless Internet Communications (WWIC), Jun 2011, Vilanova i la Geltrú, Spain. pp.150-161, 10.1007/978-3-642-21560-5_13 . hal-01583648

\section{HAL Id: hal-01583648 \\ https://inria.hal.science/hal-01583648}

Submitted on 7 Sep 2017

HAL is a multi-disciplinary open access archive for the deposit and dissemination of scientific research documents, whether they are published or not. The documents may come from teaching and research institutions in France or abroad, or from public or private research centers.
L'archive ouverte pluridisciplinaire HAL, est destinée au dépôt et à la diffusion de documents scientifiques de niveau recherche, publiés ou non, émanant des établissements d'enseignement et de recherche français ou étrangers, des laboratoires publics ou privés.

\section{(c)(1)}

Distributed under a Creative Commons Attribution| 4.0 International License 


\title{
First step in cross-layers measurement in wireless networks \\ How to adapt to resource constraints for optimizing end-to-end services?
}

\author{
Philippe Owezarski ${ }^{1,2}$, Rasha Ghassan Hasan ${ }^{1,2}$, Guillaume Kremer ${ }^{1,2}$, Pascal \\ Berthou $^{1,2 \star}$ \\ ${ }^{1}$ CNRS; LAAS; 7 avenue du colonel Roche, F-31077 Toulouse Cedex 4, France \\ ${ }^{2}$ Université de Toulouse; UPS, INSA, INP, ISAE; UT1, UTM, LAAS; F-31077 \\ Toulouse Cedex 4, France \\ e-mail: owe@laas.fr, r.g.hasan@gmail.com, gkremer@laas.fr, berthou@laas.fr
}

\begin{abstract}
Wireless networks are more and more popular, and more specifically as the access technology for all wireless devices generally used (laptops, smartphones, tablets, sensors, ...). It is then frequent to encounter at least one wireless network segment on a communication path. Because of the complexity and limits of the hertzian air medium, the end-to-end communication is impacted by the quality of such wireless networks which are not offering the same amount of resources or the same determinism in the quality of Service (QoS) level. Because of the complexity of the physical transmission mechanisms, and of the MAC layer, traffic observed at the network level can have unexpected characteristics and the network exhibits unexpected performances and QoS. At the opposite of wired network for which monitoring packets at network level is enough, monitoring wireless networks requires to look at physical, $\mathrm{MAC}$ and network layers all together and to analyze their correlation levels: this is called cross-layered monitoring in the related literature. This paper then proposes an overview of the monitoring of the quite unknown physical layers of wireless networks, and exhibits how the studies of the 3 layers are necessary for analyzing the wireless network behavior, and more generally of the end-to-end connections. As an example, this paper illustrates how the SNR impacts the delays and jitters in an unexpected way in wireless networks, thus impacting end-to-end delays and jitters.
\end{abstract}

Key words : Measurement, Cross-layering, Wireless networks, SNR, E2E services, E2E delay.

\section{Introduction}

Network communications more and more rely on wireless technologies. But compared to wired technologies, they present some limitations because of the

\footnotetext{
* This work has been partially made in the framework of the RESCUE project granted by the French National Agency for Research (ANR).
} 
hertzian support, for instance in terms of capacity limits. In addition, while the MAC layer is most of the time deterministic, and anyway quite simple, for wired network, it can be quite complex and indeterministic for wireless networks, leading to major issues for enforcing a stable performance or QoS level. In addition, because of the complexity inherent to each of the layers of significant importance in a wireless network, it is not easy to understand what happens, or to predict the service level that can be expected. For instance, interferences can impact the way the MAC layer is working, and at the end the final delivery of network packets. On the other side, a packet burst at the network level might dramatically impact the MAC performance by increasing the number of collisions, and might also induce many interferences. The convergence between wired and wireless networks and services is also of significant complexity because of differences between the technologies, as well as resources and services provided.

In such a context, connections have to cross several networks of various technologies. They therefore need to monitor the network performances and/or available resources to adapt accurately to network conditions, and getting the best possible Quality of Service (QoS). We argue that the worst conditions are almost always met in wireless networks, generally at the access point, as WLANs, GPRS, UMTS, or any other network technology used for interconnecting wireless devices (laptops, smartphones, tablets, sensors, ...) to the wired Internet infrastructure. As a consequence, the key difficulty deals with monitoring wireless networks to be encountered on Internet paths. This especially includes the very difficult task of monitoring the complex architectures of wireless networks. This is then one of the issues addressed in the framework of the French RESCUE project which aims at designing and prototyping new monitoring methodologies and tools specifically dedicated to wireless networks (WIFI, GPRS, UWB, UMTS). The originality of this work is the cross-layer monitoring approach which aims to jointly work at each of the layers involved for a wireless network, i.e. the physical, MAC and network layers. Indeed, mechanisms and protocols for each of these 3 layers are very different and then have an important impact on the way data are generated, and then on network characteristics. The idea then deals with combining and correlating measurement made and the information got from each layer in order to analyze the interdependencies that exist between the 3 layers depending on the sent traffic characteristics. By jointly analyzing the 3 layers of wireless networks altogether, it is expected to understand where and how wireless networks can experience lacks of performances and QoS. Based on such analysis results, it is also expected to find ways for adapting to wireless networks behaviors and limits for optimizing their performances and trying to guarantee the requested services.

The RESCUE project is at its very early stage. This paper then just addresses a limited scope in terms of parameters. It then focuses only on the impact of the Signal/Noise Ratio (SNR) on delays and jitters, two essential parameters for endto-end services. Despite the scope of this study is not very broad, it nevertheless has the merit to exhibit unexpected results on the level of performance and QoS provided by wireless networks depending on the air access conditions. 
The rest of this paper is organized as follows: section 2 presents the state of the art. Section 3 describes the different parameters, exclusively focusing on physic ones which are often unknown in the Internet research community. Based on this, this section also details the research problematic to be addressed in the remainder of the paper. Then section 4 starts presenting the contribution by describing the experimental platform and process. Section 5 presents the results, i.e. experiment results showing the impact of SNR on delays and jitters at network level. Finally, section 6 concludes this paper by presenting future work to be performed in the framework of the RESCUE project.

\section{State of the art in wireless networks cross-layered monitoring}

Wireless network monitoring has raised significant efforts these last years. It started with the monitoring of such wireless networks at the network (IP) level on WLANs (WIFI essentially) or on long distance Wireless networks as GPRS or UMTS [16]. This is of course of limited interest as the monitoring equipment is located at the Access Point (AP), but on the wired side. Other work, as [5], focuses on the wireless side of such networks and studies the MAC level. Another example is provided by Barrett et al. [4] which study the interaction existing between the performance of a peculiar MAC protocol and the upper-layer routing protocol used. It rigorously investigates and confirms the expectations that protocols performances at different logical layers (MAC and upper) have to be studied together. This confirms what we argued at the beginning of this paper about the need of developing cross-layered monitoring techniques for wireless networks. In particular, it has been then investigated that the physical layer has a strong impact on the performance and QoS provided by the upper layers. Let's quote a few papers illustrating this statement. Raghavendra et al. [14] analyze 802.11 traces and enlighten protocol and implementation flaws which led to problems such as packet losses. According to this paper the existence of adjacent channel leakage power, due to their small number, is as important as alien RF interferences. Verma et al. [17] present work done on a new passive method for estimating link quality based on SNR-BER mapping. It also extensively investigates the wireless cards to assess its calibration and thus ensuring correct precision. This highlights the importance of hardware to conduct RF measurements, thus pinpointing the lack of accurate monitoring tools at the wireless physical level (at least in the tools commonly used in the networking community). This is still true, despite effort by Feng et al. [11] who developed a 802.11 cross-layer measurement tool and cross-implications of layers from the application to the physical medium. This is however a good tool for whoever wants to get a grip on the cross-layer capture and analysis problematic. Sundaresan and Papagianaki [15] perfectly illustrate the need of cross-layered monitoring starting from the physical layer in wireless networks. In this paper, they investigate how clients willing to join a new 802.11 network may select the access point they want to communicate with. It more precisely compares metrics driving this choice. Contrary to previous methods, 
metrics used here get ground on cross-layer measurements; namely these metrics are the received signal strength (physical layer), a metric which characterizes the access point global throughput capacity (MAC layer) and another one which computes an estimation of response time from the AP when a client sends a request (MAC layer). They thus propose completely different methods than actual ones which are only based on the RSSI metric. In this paper, Sundaresan and Papagianaki exhibit the need to consider various parameters from any logical and physical layers. Lacage et al. [10] also highlight the fact that performances at different logical and physical layers have to be studied both singularly and plurally at the same time. They propose an extensive study of the influence of bit-rate parameters through 802.11 PHY automatic rate control over MAC protocol performances. Wang et al. [18] provide another example of the effect of PHY parameters on 802.11 MAC protocol. Many studies are also conducted to characterize the evolution of wireless medium parameters in time; for example Qiu et al.[13] and Jain et al. [7] are proposing models of interferences and wireless link behavior.

Based on this short overview on cross-layered monitoring, we want to alarm readers on the fact that most papers on wireless networks monitoring adopt the wired vision of networks and are taking aside the physical aspect of wireless communications [9]. It has to be strictly avoided, and it is understood that future tools should adopt a finer vision on what happen at the physical layer, and for the mechanisms involved at all layers of the communication stack, at the same time.

\section{Problematic}

Delay is a key parameter for characterizing and assessing the end-to-end QoS of network and distributed systems. This parameter is of real significance for users and their applications. Measured on a wired network, this dimension is quite similar at all levels of the communication architecture; it is generally measured at the network, transport or application level, but its evolution at the physical or data-link levels is pretty similar compared to upper layers. In wired networks, delays or jitters are mostly influenced by the congestion level of network devices as routers, switches, gateways, firewalls, etc.

The problematic in wireless networks is more complex. Delays and jitters are also influenced by the congestion level, but more generally, it seems that the traffic impact on the air medium is more complex, and it significantly influences the performance and QoS level of wireless networks. Depending on the traffic, it seems obvious that it can create interferences in the communication hertzian space or between adjacent communication channels, introduce noise, etc... At the physical level, it can merge some signals which are very close (the phases or periods of signals can appear the same), what makes difficult for wireless physical devices to extract the original signal. Because of that, the Physical reception algorithms and $\mathrm{MAC}$ access principle are adapted to the new traffic conditions on the air medium (i.e. degraded): the physical available throughput 
is significantly reduced, and, what we will show in the remainder of the paper, the delay is also impacted.

Physical parameters on the hertzian medium are then of key importance and must be considered and monitored for wireless networks (generally, computer scientists and engineers do not focus on so low layers requiring telecommunication skills, limiting their monitoring on layers 3 and upper (there are also some examples at layer 2 for some specific kinds of WLAN as WIFI). The rest of this section then aims at presenting the different parameters for characterizing and assessing the state of the hertzian medium for wireless communications.

The well known Signal to Noise Ratio (SNR or S/N) is the ratio between the amplitude of data signals over the amplitude of noise signals. It measures the corruption level of the original signal by noise.

A similar ratio called Signal to Noise plus Interference Ratio is an extension of the SNR. In addition of the Noise, it includes in its computing the level of interferences which are of major importance in wireless networking.

The $\frac{E_{b}}{N_{o}}$ ratio corresponds to the quantity of energy used to send one bit of data. It is also called the energy per bit over noise spectral density ratio.This value is often used as a normalization of the SNR value for a bit. Thus, the $\frac{E_{b}}{N_{o}}$ value is similar for the digital signals to the SNR for analogous signals.

The strength of the received signal is also a parameter of importance. it can be used in many applications as localization (e.g. trilateralization) [6] or link access (e.g. detection of ongoing transmissions on an emission channel) [8]. Several units exist for this parameter as the $\mathrm{mW}$ (milliWatt), the $\mathrm{dBm}$, and the Received Signal Strength Indicator or Radio Signal Strength Indication (RSSI). The three units are similar and it exists methods for converting them into each other. As the strength of the received signal does not linearly decrease with distance, but in a logarithmic way, the most used unit is the $\mathrm{dBm}$ logarithmic unit. The $\mathrm{mW}$ and $\mathrm{dBm}$ are common measurement units instead, the RSSI only exists in the context of 802.11 standards which define it along with mechanisms for measuring Radio Frequency (RF) energy by electronic circuit. Standards also specify RSSI range between 0 (no signal) and 255 (maximal signal). Nevertheless, equipment designers are not forced to use 256 different values. Atheros devices measure RSSI between 0 and 60 while for CISCO, maximal RSSI value is 100 [3]. An important note about its use is that it is defined to be used only by microcodes present on hardware devices or by drivers, but not by higher level applications. Note also that, often, drivers of wireless 802.11 chips assimilate the measure of the received signal strength with a measure of the SNR.

Adjacent Channel Power Ratio - ACPR (also called sometimes Adjacent Channel Leakage Power - ALCR) is of significant importance for communication technologies which divide the frequency band or amplitude range in several channels centered on different frequencies, e.g. OFDM. Two adjacent channels can then interfere, i.e. one sending on a channel can cause interferences on an adjacent channel (Adjacent Channel Interference - ACI). The APCR is defined as the ratio between the intensity of the energy sent on a channel over the in- 
tensity of the whole set of inference energy sent from all adjacent channels. The units generally used for computing the ACPR are $\mathrm{dBm}$, dBW or Watt.

The used bandwidth is a well known and very useful parameter measuring the width of the frequency band which contains a given percentage of the energy of a particular signal.

The Symbol Error Rate (SER) or Packet Error rate (PER) are also well known parameters defining at the receiver level the total number or erroneous symbols or packets over the total number of received symbols or packets.

The Error Vector Magnitude - EVM - measures the performance of digital communication systems. It is obtained by comparing the characteristics of the IQ modulation of a signal (signal properties in a complex space representation) with the one of a reference signal.

All the previously mentioned parameters are of significant importance for monitoring the wireless networks behaviors and performance levels. It is nevertheless not easy to make such physical measurement on the air interfaces or the hertzian medium. Such measurement needs, for being accurate, very specific and costly equipments generally used by specialists in electronics. At this stage of the RESCUE project, we are still in the study of existing equipments for performing such kinds of measurements very accurately. Thats is why in the remainder of the paper, we will focus our study on the single well known SNR parameter; many tools, and especially software and free ones, exist for measuring this parameter. The results we are presenting are not guaranteed to be accurate: they just show trends that we will check when we will have at our disposal better physical measurement equipments.

\section{Experimental platform and process}

\subsection{The test environment}

The experiment runs indoor, in the research laboratory LAAS (Laboratoire d'Analyse et d'Architecture des Systèmes) in Toulouse-France. The experiment field is a hallway inside the laboratory, rooms are located on its both sides, the walls are of concrete and the rooms furniture is mostly of wood, iron and glass. The experiment took place in a working day at the laboratory, so we can predict the existence of other experiments running at the same time, plus many users having wireless connectivity.

\subsection{The test bed}

The test bed consists of two laptops and three access points. The two laptops: PC1 with Unix OS and PC2 with Windows OS supported with Intel(R) $\mathrm{PRO} /$ Wireless card 3945ABG which is compatible with $802.11 \mathrm{a}, 802.11 \mathrm{~b}$ and $802.11 \mathrm{~g}$ wireless standards, can operate at $5 \mathrm{GHz}$ or $2.4 \mathrm{GHz}$ frequencies at speeds up to $54 \mathrm{Mbps}$. Both laptops are placed on fixed places on a mobile table which was moved to different measurement points in the hallway without changing its height $(67.5 \mathrm{~cm})$ or its direction. The three APs are $802.11 \mathrm{~g}$ ones, they 
are located as shown in figure 1 where AP1 is at the beginning of the vertical hallway, AP2 at its end and AP3 is in a lateral hallway that is orthogonal with the test hallway. All access points are at height $231(\mathrm{~cm})$ above the ground.

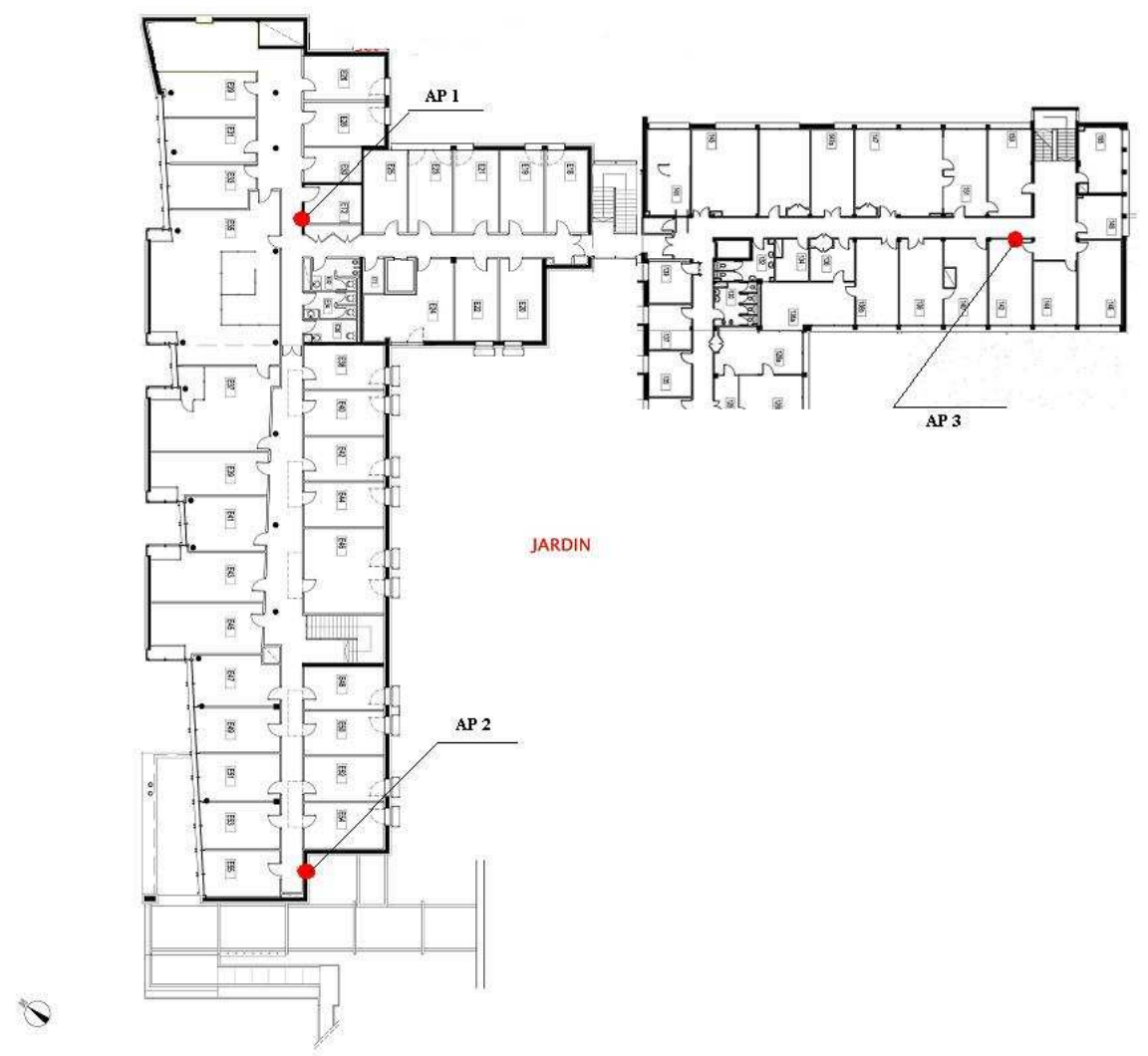

Fig. 1. Test environment: hallway in research laboratory

\subsection{The test methodology}

- Ten test points were considered in the hallway between AP1 and AP2 in a way that the first test point is located immediately under AP1, the second is further and so on. So, at each next test point we were getting further from AP1 and closer to AP2.

- The SNR at each test point was measured using NetStumbler open source measurement tool which was running on $\mathrm{PC} 2$. 
- At each test point, we were sending 200 ICMP packets from PC1 to AP1, the ACK of these packets is sent back from AP1 to PC1, this last reports the packets' history.

- Computers' clocks were synchronized via ntpdate [1] which lets access an external server and gets actual hour and date, actualizing the local computer's clock. Then through ntptrace [2] we examined the offset time in milliseconds from the external server consulted with ntpdate. We executed ntpdate and checked the offset with nptrace. This process was made several times until the offset obtained was less than 0.00008 seconds on each computer.

- Data was collected online, then analyzed offline.

\section{Results}

\subsection{Exploring SNR}

In our work, SNR was measured for the three APs at the ten test points described above. The SNR values corresponding to each test point are shown in table 1 where the values establish a minimum-maximum pattern.

\begin{tabular}{|c||c|c|c|}
\hline Test point & SNR $(\mathrm{db})$ for AP1 & SNR $(\mathrm{db})$ for AP2 & SNR $(\mathrm{db})$ for AP3 \\
\hline \hline 1 & $47-60$ & $13-37$ & $0-11$ \\
\hline 2 & $44-52$ & $11-33$ & $0-18$ \\
\hline 3 & $34-45$ & $16-39$ & $14-25$ \\
\hline 4 & $34-42$ & $0-37$ & $21-32$ \\
\hline 5 & $27-39$ & $25-39$ & $25-41$ \\
\hline 6 & $16-37$ & $23-45$ & $27-46$ \\
\hline 7 & $16-33$ & $20-44$ & $36-41$ \\
\hline 8 & $14-30$ & $31-46$ & $18-32$ \\
\hline 9 & $12-31$ & $35-57$ & $13-26$ \\
\hline 10 & $0-27$ & $50-62$ & $11-20$ \\
\hline
\end{tabular}

Table 1. Min-max SNR values of the three APs measured at the ten test points

We notice that in some ranges there are overlapped values. This overlapping is due to the natural unpredictable behavior shown by the wireless communications. This unstable behaviour derives in the possibility of obtaining different SNR measurements at the same location. For example: at test point 4, the minor SNR value for AP1 was $34(\mathrm{db})$ while at test point 5 the SNR highest value for AP1 was $39(\mathrm{db})$. In this example, we have the values in the range 34-39 (db) repeated at both points of measurements.

Figure 2 shows the SNR values range at each test point for each AP in third tile presentation to reflect as well the values distribution between the max and min SNR edges. In this figure we see that by stepping from test point 1 to test point 10 getting further from AP1 and closer to AP2, the SNR for AP1 was decreasing and for AP2 was increasing. In fact the correlation factor for SNR 
with distance for AP1 was $-0,97$ and for AP2 was $+0,98$. This lets us say that the value of SNR measured depends on the distance in a way that, the longest the distance to the AP the less the value of SNR measured is and vice versa.

Regarding SNR for AP3, we notice that between test point 1 and test point 6 it was increasing then between test point 7 and test point 10 it was decreasing. This is because while performing the experiment, we crossed the lateral corridor where AP3 is, which means that we approached it then went far from it, this resulted in making the picks of SNR values for this AP be at test point 6 .

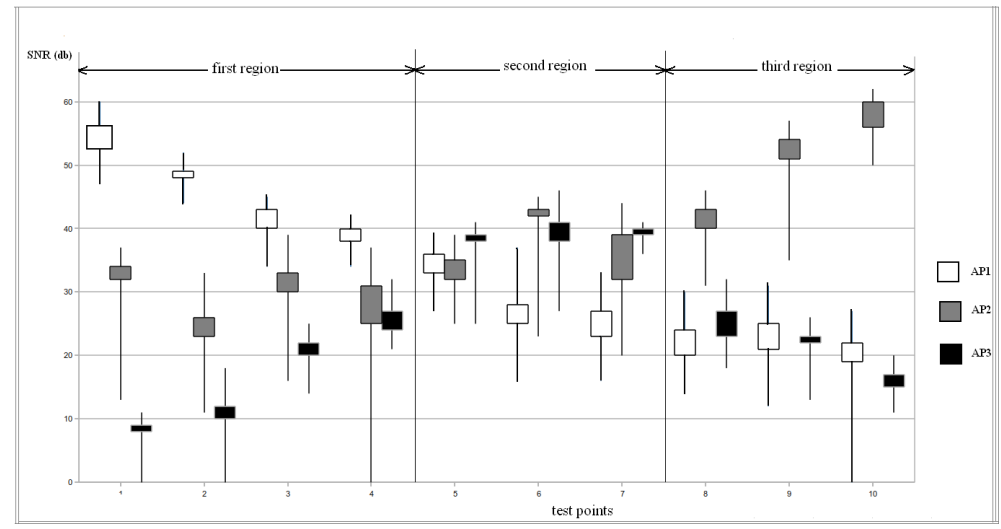

Fig. 2. SNR values in third tile representation for each AP at the ten test points

Figure 2 also illustrates that the laptop is not necessarily connected to the AP having the highest SNR. The figure can be divided into three regions according to SNR min-max values and distribution for the three APs:

1. First region: Covers 1-4 test points. AP1 SNR was the dominant. In fact, while running the experiment at those four test points, the portable computer was connected to LAAS network through AP1.

2. Second region: Covers 5-7 test points. AP3 SNR was the dominant. The portable computer was connected through AP3 at these test points while running the experiment.

3. Third region: Covers 8-10 test points. AP2 SNR was the dominant. While running the experiment at those three test points, the portable computer was connected to LAAS network through AP2.

We can say that there is a possibility that the client selects the AP of the best SNR to connect through. A better understanding of this situation can be reached by reading about roaming in the user guide of the wireless card embedded in the laptops [12] in order to know the roaming protocol that this card follows.

It is worth commenting that at test point 6; although SNR for AP2 is higher than of AP3 (figure 2), still the portable computer remained connected to AP3 
while running the experiment at this test point. The reason might be that, since the portable computer has started connecting to the network through AP3 since test point 5 , it remained connected to it at test point 6 because the SNR of AP3 at this test point was still sufficient to establish the connection even being less than the SNR of AP2.

\subsection{SNR impact on upper layers}

The goal of this study is to determin how and when do the SNR changes on the physical layer propagate to upper layers, thus affecting the E2E connection. While running the experiment we noticed that the AP signal power was much over the level needed to establish a good connection, this was reflected through the frame loss parameter wich was $0,3 \%$ (non lossy paths). This results in masking the trends. So to have a meaningful study for the SNR impact on Delay and Jitter we chose the test point at which the average SNR value was the minimum, it was test point 3. We considered the time stamps for SNR values as a reference and shifted the Delay and Jitter traces with several time values up to a maximum shift of 1 second on millisecond scale. Table 2 shows the correlation values at each time shift.

\begin{tabular}{|c|c|c|}
\hline Time shift $(\mathrm{ms})$ & Corr(SNR,Delay) & Corr(SNR,Jitter) \\
\hline \hline 0 & 0,89 & 0,67 \\
\hline 100 & 0,84 & 0,76 \\
\hline 200 & 0,61 & 0,48 \\
\hline 300 & $-0,88$ & $-0,81$ \\
\hline 400 & $-0,69$ & $-0,07$ \\
\hline 500 & $-0,37$ & 0,49 \\
\hline 600 & 0,21 & 0,14 \\
\hline 700 & 0,46 & 0,6 \\
\hline 800 & 0,25 & $-0,01$ \\
\hline 900 & 0,59 & 0,83 \\
\hline
\end{tabular}

Table 2. Correlation values for SNR with Delay and Jitter at test point 3

We notice that at time shift $300 \mathrm{~ms}$, the impact of SNR on other both parameters appeared in a way that the decrease in SNR reflects in Delay and Jitter increase and vice versa. In other words, the decrease of SNR on the physical layer propagates to upper layers in term of Delay and Jitter increase. In our experiment the propagation happened with time delay of $300 \mathrm{~ms}$ where the correlation values were $-0,88$ and $-0,81$ respectively.

On the other hand, we notice that a small change in SNR values is strongly reflected on upper layers. For example, while running the experiment, an increase in SNR value from $43 \mathrm{db}$ to $45 \mathrm{db}$ made the Delay value falls from $124 \mathrm{~ms}$ to 59 ms after time shift of $300 \mathrm{~ms}$, and a decrease in SNR value from $45 \mathrm{db}$ to $44 \mathrm{db}$ resulted in Delay increase from $3 \mathrm{~ms}$ to $91 \mathrm{~ms}$. 


\section{Conclusion}

This paper aims at motivating the use of cross-layered monitoring in wireless networks. For this purpose, it puts emphasis on the complexity of the largely unknown (in the networking community) physical layer and all its constraints. The paper then makes a brief but significant presentation of the different parameters to be measured at the physical layer. It exhibits the inherent complexity of such parameters and emphasizes on the difficulty of accurately measuring them. A strong effort has to be provided to find suited monitoring equipments (we are currently taking advantage of the equipments own by researchers in electronics in our lab) or design and develop new ones. The paper then presents a very preliminary and illustrative study aiming at exhibiting the unexpected behaviors of the physical layers of wireless networks. It is shown that the SNR has a significant impact on the communication delay and jitter in a completely unexpected way in terms of intensity: a small change in the SNR causes huge changes in delay and jitters values. This is especially damageable for the global network performance, as well as for streaming or real-time applications which require stable jitter and low delays. It then appears clearly that the current evolution of wireless networks, especially devoted for favoring the integration of digital applications on wireless supports, is not completely fulfilling its objectives because of such unexpected sensitivity to SNR.

\section{References}

1. ntpdate, set the date and time via ntp. In: http://www.eecis.udel.edu

2. ntptrace, trace a chain of ntp servers back to the primary source. In: http://www.eecis.udel.edu

3. Bardwell, J.: Converting Signal Strength Percentage to dBm Values (Nov 2002), http://www.wildpackets.com/elements/whitepapers/Converting_Signal_Strength.pdf

4. Barrett, C., Drozda, M., Marathe, A., Marathe, M.: Analyzing interaction between network protocols, topology and traffic in wireless radio networks. In: Wireless Communications and Networking, 2003. WCNC 2003. 2003 IEEE. vol. 3, pp. 1760 -1766 vol.3 (2003)

5. Claveirole, T., de AmorimMarcelo, D.: Wipal and wscout, two hands-on tools for wireless packet traces manipulation and visualization. In: ACM Mobicom Workshop on Wireless Network Testbeds, Experimental Evaluation, and Characterization. MobiCom '08 (2008)

6. Hightower, J., Vakili, C., Borriello, G., Want, R.: Design and Calibration of the SpotON Ad-Hoc Location Sensing System (2001), http://citeseerx.ist.psu.edu/viewdoc/summary?doi=10.1.1.23.6231

7. Jain, K., Padhye, J., Padmanabhan, V.N., Qiu, L.: Impact of interference on multihop wireless network performance. In: Proceedings of the 9th annual international conference on Mobile computing and networking. pp. 66-80. MobiCom '03, ACM, New York, NY, USA (2003), http://doi.acm.org/10.1145/938985.938993

8. Kolar, V., Bharath, K., Abu-Ghazaleh, N.B., Riihijarvi, J.: Contention in multihop wireless networks: model and fairness analysis. In: Proceedings of the 12th ACM international conference on Modeling, analysis and simulation of wireless 
and mobile systems. pp. 21-29. MSWiM '09, ACM, New York, NY, USA (2009), http://doi.acm.org/10.1145/1641804.1641812

9. Kotz, D., Newport, C., Elliott, C.: The mistaken axioms of wireless-network research. Tech. rep., Dartmouth College (Jul 2003)

10. Lacage, M., Manshaei, M.H., Turletti, T.: "ieee 802.11" rate adaptation: a practical approach. In: Proceedings of the 7th ACM international symposium on Modeling, analysis and simulation of wireless and mobile systems. pp. 126-134. MSWiM '04, ACM, New York, NY, USA (2004), http://doi.acm.org/10.1145/1023663.1023687

11. Li, F., Li, M., Lu, R., Wu, H., Claypool, M., Kinicki, R.: Tools and techniques for measurement of ieee 802.11 wireless networks. In: Modeling and Optimization in Mobile, Ad Hoc and Wireless Networks, 2006 4th International Symposium on. pp. $1-8(2006)$

12. Parkway, E.Y., Hillsboro: Intel(r) pro/wireless 3945abg network connection user guide. In: User Guide [Online]. Available: ftp://download.intel.com/support/wireless/wlan/sb/3945abgug.pdf. Intel Corporation (2004-2005)

13. Qiu, L., Zhang, Y., Wang, F., Han, M.K., Mahajan, R.: A general model of wireless interference. In: Proceedings of the 13th annual ACM international conference on Mobile computing and networking. pp. 171-182. MobiCom '07, ACM, New York, NY, USA (2007), http://doi.acm.org/10.1145/1287853.1287874

14. Raghavendra, R., Belding, E., Papagiannaki, K., Almeroth, K.: Unwanted link layer traffic in large ieee 802.11 wireless networks. Mobile Computing, IEEE Transactions on 9(9), $1212-1225$ (2010)

15. Sundaresan, K., Papagiannaki, K.: The need for cross-layer information in access point selection algorithms. In: Proceedings of the 6th ACM SIGCOMM conference on Internet measurement. pp. 257-262. IMC '06, ACM, New York, NY, USA (2006), http://doi.acm.org/10.1145/1177080.1177113

16. Svoboda, P., Ricciato, F., Hasenleithner, E., Pilz, R.: Composition of gprs/umts traffic : snapshots from a live network. In: 4th Int'l Workshop on Internet Performance, Simulation, Monitoring and Measurement. IPS-MOME'06 (2006)

17. Verma, L., Kim, S., Choi, S., Lee, S.J.: Reliable, low overhead link quality estimation for 802.11 wireless mesh networks. In: Sensor, Mesh and Ad Hoc Communications and Networks Workshops, 2008. SECON Workshops '08. 5th IEEE Annual Communications Society Conference on. pp. 1 -6 (2008)

18. Wang, L.C., Chen, A., Huang, S.Y.: A cross-layer investigation for the throughput performance of csma/ca-based wlans with directional antennas and capture effect. Vehicular Technology, IEEE Transactions on 56(5), 2756 -2766 (2007) 